\title{
ASYMPTOTIC IMPROVEMENTS TO THE LOWER BOUND OF CERTAIN BIPARTITE TURÁN NUMBERS
}

\author{
SIMEON BALL AND VALENTINA PEPE
}

\begin{abstract}
We show that there are graphs with $n$ vertices containing no $K_{5,5}$ which have about $\frac{1}{2} n^{7 / 4}$ edges, thus proving that $e x\left(n, K_{5,5}\right) \geq \frac{1}{2}(1+o(1)) n^{7 / 4}$. This bound gives an asymptotic improvement to the known lower bounds on ex $\left(n, K_{t, s}\right)$ for $t=5$ when $5 \leq s \leq 12$, and $t=6$ when $6 \leq s \leq 8$.
\end{abstract}

\section{INTRODUCTION}

Let $H$ be a fixed graph. The Turán number of $H$, denoted ex $(n, H)$, is the maximum number of edges in a graph on $n$ vertices which contains no copy of $H$. The Erdös-Stone Theorem from [7] gives an asymptotic formula for the Turán number of any non-bipartite graph, and this formula depends on the chromatic number of the graph $H$.

When $H$ is a complete bipartite graph, determining the Turán number is related to the "Zarankiewicz problem" (see [3], Chap. VI, Sect.2, and [9] for more details and references). In many cases even the question of determining the right order of magnitude for $e x(n, H)$ is not known.

Let $K_{t, s}$ denote the complete bipartite graph with $t$ vertices in one class and $s$ vertices in the other. Kövari, Sós and Turán [10] proved that for $s \geq t$

$$
e x\left(n, K_{t, s}\right) \leq \frac{1}{2}(s-1)^{1 / t} n^{2-1 / t}+\frac{1}{2}(t-1) n .
$$

The best known general lower bounds, obtained by probabilistic constructions, are

$$
e x\left(n, K_{t, s}\right)=\Omega\left(n^{2-(s+t-2) /(s t-1)}\right),
$$

see Erdős and Spencer [6], and

$$
e x\left(n, K_{t, t}\right)=\Omega\left((\log n)^{1 /\left(t^{2}-1\right)} n^{2-(2 /(t+1))}\right),
$$

see Bohman and Keevash [2].

The upper bound was shown to be asymptotically tight for $s \geq t=2$ (Erdös, Rényi and Sós [5], Brown [4] for $s=t=2$, Füredi [9] for $s \geq t=2$ ). Füredi [8] improved on the

Date: 3 September 2011.

The first author acknowledges the support of the project MTM2008-06620-C03-01 of the Spanish Ministry of Science and Education and the project 2009-SGR-01387 of the Catalan Research Council. 
upper bound (1.1) proving that

$$
e x\left(n, K_{3,3}\right)=\frac{1}{2} n^{5 / 3}+o\left(n^{5 / 3}\right),
$$

for which Brown's construction from [4] gives the lower bound.

Alon, Rónyai and Szabó [1] showed, by construction, that if $s \geq(t-1)$ ! +1 then

$$
e x\left(n, K_{t, s}\right) \geq \frac{1}{2}(1+o(1)) d_{t}(s-1)^{1 / t} n^{2-1 / t},
$$

where $d_{t}$ is some constant.

The first open case for which the asymptotic behaviour of $e x\left(n, K_{t, s}\right)$ is not known is $K_{4,4}$. The probabilistic lower bound gives ex $\left(n, K_{4,4}\right) \geq c n^{8 / 5}+o\left(n^{8 / 5}\right)$, but Brown's bound for $e x\left(n, K_{3,3}\right)$ implies $e x\left(n, K_{4,4}\right) \geq \frac{1}{2} n^{5 / 3}+o\left(n^{5 / 3}\right)$. The upper bound (1.1) gives $\operatorname{ex}\left(n, K_{4,4}\right) \leq c n^{7 / 4}+o\left(n^{7 / 4}\right)$.

The upper bound (1.1) for $K_{5,5}$ gives $e x\left(n, K_{5,5}\right) \leq c n^{9 / 5}+o\left(n^{9 / 5}\right)$, whereas the probabilistic lower bound for $K_{5,5}$ gives $e x\left(n, K_{5,5}\right) \geq c n^{5 / 3}+o\left(n^{5 / 3}\right)$. In this article we shall show that the graphs, considered by Alon, Rónyai and Szabó in [1], which contain no $K_{4,7}$ in fact contain no $K_{5,5}$, thus proving that

$$
e x\left(n, K_{5,5}\right) \geq \frac{1}{2}(1+o(1)) n^{7 / 4} .
$$

This gives an asymptotic improvement to the lower bounds of $e x\left(n, K_{5, s}\right)$ for $5 \leq s \leq 12$ and $e x\left(n, K_{6, s}\right)$ for $6 \leq s \leq 8$.

\section{THE NORM GRAPH}

Suppose that $q=p^{h}$, where $p$ is a prime, and denote by $\mathbb{F}_{q}$ the finite field with $q$ elements. We will use the following properties of finite fields. For any $a, b \in \mathbb{F}_{q},(a+b)^{p^{i}}=a^{p^{i}}+b^{p^{i}}$, for any $i \in \mathbb{N}$. Note that $(a-b)^{p^{i}}=a^{p^{i}}-b^{p^{i}}$, since either $p^{i}$ is odd or $-1=1$. Secondly, for all $a \in \mathbb{F}_{q^{i}}, a^{q}=a$ if and only if $a \in \mathbb{F}_{q}$. Finally $a^{q^{2}+q+1} \in \mathbb{F}_{q}$, for all $a \in \mathbb{F}_{q^{3}}$, since $a^{q^{3}}=a$.

Let $\Gamma$ be the graph with vertices $(a, \alpha) \in \mathbb{F}_{q^{3}} \times \mathbb{F}_{q}, \alpha \neq 0$, where $(a, \alpha)$ is joined to $\left(a^{\prime}, \alpha^{\prime}\right)$ if and only if $\left(a+a^{\prime}\right)^{q^{2}+q+1}=\alpha \alpha^{\prime}$. In [1] Alon, Rónyai and Szabó prove that $\Gamma$ contains no $K_{4,7}$, our aim here is to show that it also contains no $K_{5,5}$.

Let

$$
V=\left\{\left(1, a, a^{q}, a^{q^{2}}, a^{q+1}, a^{q^{2}+1}, a^{q^{2}+q}, a^{q^{2}+q+1}, 0\right) \mid a \in \mathbb{F}_{q^{3}}\right\} \subset \mathbb{F}_{q^{3}}^{9} .
$$

Let $b$ be the symmetric bilinear form on $\mathbb{F}_{q^{3}}^{9}$ defined by

$$
b(x, y)=\sum_{i=1}^{8} x_{i} y_{9-i}-x_{9} y_{9}
$$


Let $\perp$ be defined in the usual way, so that given $S \subset \mathbb{F}_{q^{3}}^{9}$,

$$
S^{\perp}=\left\{y \in \mathbb{F}_{q^{3}}^{9} \mid b(x, y)=0, \text { for all } x \in S\right\} .
$$

We wish to define the same graph $\Gamma$, so that adjacency is given by the bilinear form. Consider the graph $\Gamma^{\prime}$ with vertex set the set of vectors $x=v+\alpha e_{9}$, where $e_{9}=$ $(0,0,0,0,0,0,0,0,1), v \in V$ and $\alpha \in \mathbb{F}_{q}, \alpha \neq 0$, and where two vertices $x=v+\alpha e_{9}$ and $x^{\prime}=v^{\prime}+\alpha^{\prime} e_{9}$ are adjacent if and only if $b\left(x, x^{\prime}\right)=0$. It is a simple matter to verify that the graph $\Gamma^{\prime}$ is isomorphic to the graph $\Gamma$; we shall call it $\Gamma$ from now on.

For any subset $S$ of the vertices the common neighbours $x$ of $S$ satisfy $b(x, w)=0$ for all $w \in S$ which, by linearity, is the condition $b(x, w)=0$ for all $w \in\langle S\rangle$. Importantly, this implies that the common neighbours of the vertices in $S$ (the vertices in $S^{\perp}$ ) are common neighbours of all the vertices in $\langle S\rangle$.

If $S$ contains two vectors of the form $v+\alpha e_{9}$ and $v+\alpha^{\prime} e_{9}$ for some $v \in V$, then $e_{9} \in\langle S\rangle$ and the vertices of $S$ have no common neighbours, since $\left\{e_{9}\right\}^{\perp}$ is the hyperplane defined by the equation $x_{9}=0$ and $x_{9} \neq 0$ for any vertex of $\Gamma$.

Throughout the article dim will refer to vector space dimension.

The following lemma is a special case of [11, Theorem 3]. We include a proof here for the sake of completeness.

Lemma 2.1. If $|S| \geq 4$ and $e_{9} \notin\langle S\rangle$ then $\operatorname{dim}(\langle S\rangle) \geq 4$.

Proof. Let $M$ be the $4 \times 8$ matrix whose $i$-th row is $\left(1, a_{i}, a_{i}^{q}, a_{i}^{q^{2}}, a_{i}^{q+1}, a_{i}^{q^{2}+1}, a_{i}^{q^{2}+q}, a_{i}^{q^{2}+q+1}\right)$, where $\left(1, a_{i}, a_{i}^{q}, a_{i}^{q^{2}}, a_{i}^{q+1}, a_{i}^{q^{2}+1}, a_{i}^{q^{2}+q}, a_{i}^{q^{2}+q+1}, \alpha\right) \in S$, and in which we can assume that $a_{i}$ are pairwise distinct since $e_{9} \notin\langle S\rangle$. It suffices to prove that $\operatorname{rank}(M) \geq 4$ since $\operatorname{dim}(\langle S\rangle) \geq \operatorname{rank}(M)$.

By elementary column operations $\operatorname{rank}(M)=\operatorname{rank}\left(M^{*}\right)$, where $M^{*}$ is the $4 \times 8$ matrix whose first row is $(1,0,0,0,0,0,0,0)$ and whose other rows are $\left(1, a_{i}-a_{1},\left(a_{i}-a_{1}\right)^{q},\left(a_{i}-\right.\right.$ $\left.\left.a_{1}\right)^{q^{2}},\left(a_{i}-a_{1}\right)^{q+1},\left(a_{i}-a_{1}\right)^{q^{2}+1},\left(a_{i}-a_{1}\right)^{q^{2}+q},\left(a_{i}-a_{1}\right)^{q^{2}+q+1}\right)$. We start by making the eighth column of $M^{*}$ and then the seventh, sixth, etc, in the following way. For example, to make the fifth column we add $a_{1}^{q+1}$ times the first column, subtract $a_{1}^{q}$ times the second column and subtract $a_{1}$ times the third column giving $a_{i}^{q+1}-a_{1} a_{i}^{q}-a_{1}^{q} a_{i}+a_{1}^{q+1}=\left(a_{i}-a_{1}\right)^{q+1}$.

Considering the second, fifth, sixth and eighth columns of $M^{*}$, and dividing the $i$-th row by $a_{i}-a_{1},(i=2,3,4)$, we have that, $\operatorname{rank}(M) \geq 1+\operatorname{rank}\left(M^{\prime}\right)$, where $M^{\prime}$ is the $3 \times 4$ matrix whose $i$-th row is $\left(1, b_{i}, b_{i}^{q}, b_{i}^{q+1}\right)$, where $b_{i}=\left(a_{i+1}-a_{1}\right)^{q}$. Since $x \mapsto x^{q}$ is a bijection of $\mathbb{F}_{q^{3}}$, the $b_{i}$ are pairwise distinct.

By elementary column operations $\operatorname{rank}\left(M^{\prime}\right)=\operatorname{rank}\left(M^{* *}\right)$ where $M^{*}$ is the $3 \times 4$ matrix whose first row is $(1,0,0,0)$ and whose other rows are $\left(1, b_{i}-b_{1},\left(b_{i}-b_{1}\right)^{q},\left(b_{i}-b_{1}\right)^{q+1}\right)$. Just considering the second and fourth columns, and dividing the $i$-th row by $b_{i}-b_{1}$, $(i=2,3)$, we have that, $\operatorname{rank}\left(M^{\prime}\right) \geq 1+\operatorname{rank}\left(M^{\prime \prime}\right)$, where $M^{\prime \prime}$ is the $2 \times 2$ matrix whose 
$i$-th row is $\left(1, c_{i}\right)$, where $c_{i}=\left(b_{i+1}-b_{1}\right)^{q}$. Since $x \mapsto x^{q}$ is a bijection of $\mathbb{F}_{q^{3}}, c_{1} \neq c_{2}$, and so $M^{\prime \prime}$ has rank 2 . Hence, $M$ has rank 4.

Define a subset of the projective space $\mathrm{PG}\left(8, q^{3}\right)$ by

$$
V^{*}=\left\{\left\langle\left(1, a, a^{q}, a^{q^{2}}, a^{q+1}, a^{q^{2}+1}, a^{q^{2}+q}, a^{q^{2}+q+1}, 0\right)\right\rangle \mid a \in \mathbb{F}_{q^{3}}\right\} \cup\left\{\left\langle e_{8}\right\rangle\right\},
$$

where $e_{8}=(0,0,0,0,0,0,0,1,0)$.

LEMMA 2.2. There is a group of linear automorphisms of $\mathbb{F}_{q^{3}}^{9}$ that induces a 3-transitive action on $V^{*}$.

Proof. Consider the group of endomorphisms of $\mathbb{F}_{q^{3}}^{9}$ generated by

$$
\sigma\left(\left(x_{1}, \ldots, x_{8}, x_{9}\right)\right)=\left(x_{8}, x_{7}, x_{6}, x_{5}, x_{4}, x_{3}, x_{2}, x_{1}, x_{9}\right)
$$

and for each $\lambda \in \mathbb{F}_{q^{3}}$,

$$
\begin{gathered}
\tau_{\lambda}\left(\left(x_{1}, \ldots, x_{8}, x_{9}\right)\right)=\left(x_{1}, x_{2}+\lambda x_{1}, x_{3}+\lambda^{q} x_{1}, x_{4}+\lambda^{q^{2}} x_{1}, x_{5}+\lambda x_{3}+\lambda^{q} x_{2}+\lambda^{q+1} x_{1},\right. \\
x_{6}+\lambda x_{4}+\lambda^{q^{2}} x_{2}+\lambda^{q^{2}+1} x_{1}, x_{7}+\lambda^{q} x_{4}+\lambda^{q^{2}} x_{3}+\lambda^{q^{2}+q} x_{1}, \\
\left.x_{8}+\lambda x_{7}+\lambda^{q} x_{6}+\lambda^{q^{2}} x_{5}+\lambda^{q+1} x_{4}+\lambda^{q^{2}+1} x_{3}+\lambda^{q^{2}+q} x_{2}+\lambda^{q^{2}+q+1} x_{1}, x_{9}\right)
\end{gathered}
$$

and

$$
\alpha_{\lambda}\left(\left(x_{1}, \ldots, x_{8}, x_{9}\right)\right)=\left(x_{1}, \lambda x_{2}, \lambda^{q} x_{3}, \lambda^{q^{2}} x_{4}, \lambda^{q+1} x_{5}, \lambda^{q^{2}+1} x_{6}, \lambda^{q^{2}+q} x_{7}, \lambda^{q^{2}+q+1} x_{8}, x_{9}\right) .
$$

These linear maps are all automorphisms of $V^{*}$ and act transitively. Indeed, if we write $\bar{a}=\left\langle\left(1, a, a^{q}, a^{q^{2}}, a^{q+1}, a^{q^{2}+1}, a^{q^{2}+q}, a^{q^{2}+q+1}, 0\right)\right\rangle$ then $\sigma(\bar{a})=\overline{a^{-1}}, a \neq 0, \sigma(\overline{0})=\left\langle e_{8}\right\rangle$, $\sigma\left(\left\langle e_{8}\right\rangle\right)=\overline{0}, \tau_{\lambda}(\bar{a})=\overline{a+\lambda}$ and $\alpha_{\lambda}(\bar{a})=\overline{\lambda a}$.

Moreover, the automorphisms $\tau_{\lambda}$ fix $\left\langle e_{8}\right\rangle$ and act transitively on the remaining points. The automorphisms $\alpha_{\lambda}$ fix $\left\langle e_{8}\right\rangle$ and $\langle\overline{0}\rangle$ and act transitively on the remaining points. Thus, the action is 3 -transitive.

We note that the group in Lemma 2.2 is isomorphic to $\operatorname{PGL}\left(2, q^{3}\right)$.

LEMma 2.3. For any 4-dimensional subspace $U$ of $\mathbb{F}_{q^{3}}^{9}$ either $|U \cap V| \leq 4$ or $|U \cap V| \geq q$.

Proof. Let us suppose that $|U \cap V| \geq 5$. Thus $U^{*}=\{\langle u\rangle \mid u \in U\}$ has the property that $\left|U^{*} \cap V^{*}\right| \geq 5$, since $V$ intersects any 1-dimensional subspace in at most one vector.

By Lemma 2.2, we can assume that four of the points in this intersection are $\left\langle v_{1}\right\rangle,\left\langle v_{2}\right\rangle$, $\left\langle v_{3}\right\rangle$ and $\left\langle v_{4}\right\rangle$, with $v_{1}=(0, \ldots, 0,1,0), v_{2}=(1,0, \ldots, 0), v_{3}=(1, \ldots, 1,0)$ and $v_{4}=$ $\left(1, a, a^{q}, a^{q^{2}}, a^{q+1}, a^{q^{2}+1}, a^{q^{2}+q}, a^{q^{2}+q+1}, 0\right)$ for some fixed $a \neq 0,1$. 
Since $\operatorname{dim} U=4$ the fifth point in this intersection $\left\langle v_{5}\right\rangle$, where $v_{5}=\left(1, b, b^{q}, b^{q^{2}}, b^{q+1}, b^{q^{2}+1}, b^{q^{2}+q}, b^{q^{2}+q+1}, 0\right)$ for some $b \neq 0,1, a$, is a linear combination of these 4 vectors. Therefore, there are $\lambda_{1}, \lambda_{2}, \lambda_{3}, \lambda_{4} \in \mathbb{F}_{q^{3}}$ for which

$$
\left(1, b, b^{q}, b^{q^{2}}, b^{q+1}, b^{q^{2}+1}, b^{q^{2}+q}, b^{q^{2}+q+1}, 0\right)=\sum_{i=1}^{4} \lambda_{i} v_{i}
$$

If $\lambda_{4}=0$ then the second, third and fifth coordinates give $\lambda_{3}=b, \lambda_{3}=b^{q}$ and $\lambda_{3}=b^{q+1}$, which imply $\lambda_{3}^{2}=\lambda_{3}=b$, a contradiction since $b \neq 0,1$. If $\lambda_{3}=0$ then the second, third and fifth coordinates give $\lambda_{4} a=b, \lambda_{4} a^{q}=b^{q}$ and $\lambda_{4} a^{q+1}=b^{q+1}$, which imply $\lambda_{4}^{2}=\lambda_{4}=b / a$, a contradiction since $b \neq 0, a$. Hence, we can assume that $\lambda_{3} \lambda_{4} \neq 0$.

Considering the second, third and fourth coordinates we have $b=\lambda_{3}+\lambda_{4} a, b^{q}=\lambda_{3}+\lambda_{4} a^{q}$ and $b^{q^{2}}=\lambda_{3}+\lambda_{4} a^{q^{2}}$ which give $b-b^{q}=\left(a-a^{q}\right) \lambda_{4}$ and $b^{q^{2}}-b=\left(a^{q^{2}}-a\right) \lambda_{4}$. Applying the map $x \mapsto x^{q}$ to the latter equation gives $b-b^{q}=\left(a-a^{q}\right) \lambda_{4}^{q}$ and so $0=\left(a-a^{q}\right)\left(\lambda_{4}-\lambda_{4}^{q}\right)$.

If $a \notin \mathbb{F}_{q}$ then $\lambda_{4} \in \mathbb{F}_{q}$. Now applying the map $x \mapsto x^{q}$ to $b=\lambda_{3}+\lambda_{4} a$, we have $b^{q}=\lambda_{3}^{q}+\lambda_{4} a^{q}$ and combining this with $b^{q}=\lambda_{3}+\lambda_{4} a^{q}$ gives $\lambda_{3} \in \mathbb{F}_{q}$. The second and seventh coordinates give $b=\lambda_{3}+\lambda_{4} a, b^{q^{2}+q}=\lambda_{3}+\lambda_{4} a^{q^{2}+q}$ and so $b^{q^{2}+q+1}=\left(\lambda_{3}+\right.$ $\left.\lambda_{4} a\right)\left(\lambda_{3}+\lambda_{4} a^{q^{2}+q}\right) \in \mathbb{F}_{q}$. Since $a^{q^{2}+q+1} \in \mathbb{F}_{q}$ and $\lambda_{3} \lambda_{4} \neq 0$ this implies $a^{q^{2}+q}+a \in \mathbb{F}_{q}$. Thus, $a^{q^{2}+q}+a=a^{q^{2}+1}+a^{q}$, which gives $\left(a^{q}-a\right)\left(a^{q^{2}}-1\right)=0$ and so $a \in \mathbb{F}_{q}$, a contradiction.

Therefore $a \in \mathbb{F}_{q}$ and for each $b \in \mathbb{F}_{q}$, the vector $\left(1, b, b, b, b^{2}, b^{2}, b^{2}, b^{3}, 0\right)$ is an $\mathbb{F}_{q}$-linear combination of $v_{1}, v_{2}, v_{3}$ and $v_{4}$. This implies $\left|U^{*} \cap V^{*}\right| \geq q+1$. Now going back to the vector space, noting that $e_{8} \notin V$, we have $|U \cap V| \geq q$.

TheOrem 2.4. For $q \geq 7$ the graph $\Gamma$ contains no $K_{5,5}$.

Proof. Let $S$ be a set of 5 vertices of $\Gamma$.

If $S$ contains two vectors of the form $v+\alpha e_{9}$ and $v+\alpha^{\prime} e_{9}$ for some $v \in V$, then $e_{9} \in\langle S\rangle$ and the vertices of $S$ have no common neighbours, since $\left\{e_{9}\right\}^{\perp}$ is the hyperplane $H$ defined by the equation $x_{9}=0$, and all vertices of $\Gamma$ have $x_{9} \neq 0$.

Therefore, suppose that $e_{9} \notin\langle S\rangle$. By Lemma 2.1, we have that $\operatorname{dim}(\langle S\rangle) \geq 4$. Moreover, we can suppose that $e_{9} \notin S^{\perp}$ since $e_{9} \in S^{\perp}$ implies $S \subset H$, which it is not.

If $\operatorname{dim}(\langle S\rangle)=4$ then consider $U=\left\langle S, e_{9}\right\rangle \cap H$. The subspace $U$ is 4-dimensional and contains at least 5 vectors of $V$ and so by Lemma 2.3 it contains at least $q$ vectors of $V$. For each $u \in U \cap V$, there exists an $\alpha \in \mathbb{F}_{q^{3}}$ such that $u+\alpha e_{9} \in\langle S\rangle$. We want to prove that $\alpha \in \mathbb{F}_{q}, \alpha \neq 0$, and hence conclude that $u+\alpha e_{9}$ is a vertex of $\Gamma$. We can assume that there are two vertices $u^{\prime}+\alpha^{\prime} e_{9}, u^{\prime \prime}+\alpha^{\prime \prime} e_{9} \in S^{\perp}$, since otherwise the vertices in $\mathrm{S}$ have at most one common neighbour. Note that $\alpha^{\prime}, \alpha^{\prime \prime} \in \mathbb{F}_{q}, \alpha^{\prime}, \alpha^{\prime \prime} \neq 0, u^{\prime}, u^{\prime \prime} \in V$, and $u^{\prime} \neq u^{\prime \prime}$ since $e_{9} \notin S^{\perp}$. Now $u+\alpha e_{9} \in S$ and $u^{\prime}+\alpha^{\prime} e_{9} \in S^{\perp}$ implies

$$
b\left(u+\alpha e_{9}, u^{\prime}+\alpha^{\prime} e_{9}\right)=(a+b)^{q^{2}+q+1}-\alpha \alpha^{\prime}=0,
$$


where

$$
u=\left(1, a, a^{q}, a^{q^{2}}, a^{q+1}, a^{q^{2}+1}, a^{q^{2}+q}, a^{q^{2}+q+1}, 0\right)
$$

and

$$
u^{\prime}=\left(1, b, b^{q}, b^{q^{2}}, b^{q+1}, b^{q^{2}+1}, b^{q^{2}+q}, b^{q^{2}+q+1}, 0\right) .
$$

Since $\alpha^{\prime} \in \mathbb{F}_{q}, \alpha^{\prime} \neq 0$, we can conclude that $\alpha \in \mathbb{F}_{q}$. If $\alpha=0$ then $b=-a$ and so if we repeat the above replacing $u^{\prime}+\alpha^{\prime} e_{9}$ with $u^{\prime \prime}+\alpha^{\prime \prime} e_{9}$ we have that $u^{\prime}=u^{\prime \prime}$, a contradiction. Thus $\alpha \in \mathbb{F}_{q}, \alpha \neq 0$, and $u+\alpha e_{9}$ is a vertex of $\Gamma$. This implies that $\langle S\rangle$ contains at least $q$ vertices of $\Gamma$. As mentioned before, the common neighbours of the vertices in $S$ (the vertices in $S^{\perp}$ ) are common neighbours of all the vertices in $\langle S\rangle$. In [1] Alon, Rónyai and Szabó prove that $\Gamma$ contains no $K_{4,7}$, so $S^{\perp}$ contains at most 3 vertices of the graph, hence the five vertices of $S$ have at most 3 common neighbours.

If $\operatorname{dim}(\langle S\rangle)=5$ then, since $b$ is non-degenerate, $\operatorname{dim} S^{\perp}=4$. The subspace $U=\left\langle S^{\perp}, e_{9}\right\rangle \cap$ $H$ is 4 -dimensional and so by Lemma 2.3 contains at most 4 vectors of $V$ or at least $q$. If $|U \cap V| \leq 4$ then $S^{\perp}$ contains at most 4 vertices of $\Gamma$, since $e_{9} \notin S^{\perp}$, and so the vertices in $S$ have at most 4 common neighbours. Finally, consider the case $|U \cap V| \geq q$. For each $u \in U \cap V$, there exists an $\alpha \in \mathbb{F}_{q^{3}}$ such that $u+\alpha e_{9} \in S^{\perp}$. We want to prove that $\alpha \in \mathbb{F}_{q}$, $\alpha \neq 0$, and hence conclude that $u+\alpha e_{9}$ is a vertex of $\Gamma$. For each vertex $u^{\prime}+\alpha^{\prime} e_{9} \in S$

$$
b\left(u+\alpha e_{9}, u^{\prime}+\alpha^{\prime} e_{9}\right)=(a+b)^{q^{2}+q+1}-\alpha \alpha^{\prime}=0,
$$

where

$$
u=\left(1, a, a^{q}, a^{q^{2}}, a^{q+1}, a^{q^{2}+1}, a^{q^{2}+q}, a^{q^{2}+q+1}, 0\right)
$$

and

$$
u^{\prime}=\left(1, b, b^{q}, b^{q^{2}}, b^{q+1}, b^{q^{2}+1}, b^{q^{2}+q}, b^{q^{2}+q+1}, 0\right) .
$$

Since $\alpha^{\prime} \in \mathbb{F}_{q}, \alpha^{\prime} \neq 0$, we can conclude that $\alpha \in \mathbb{F}_{q}$. If $\alpha=0$ then $b=-a$ and so for each vertex $v+\beta e_{9}$ in $S, v=u^{\prime}$, which is a contradiction since $e_{9} \notin\langle S\rangle$. Thus $\alpha \in \mathbb{F}_{q}, \alpha \neq 0$, and $u+\alpha e_{9}$ is a vertex of $\Gamma$. Therefore, $S^{\perp}$ contains at least $q$ vertices of $\Gamma$ and so the vertices in $S$ have at least $q$ common neighbours. However, this implies that $\Gamma$ contains a $K_{5,7}$ and therefore a $K_{4,7}$, which is not the case.

\section{ACKnowledgements}

We sincerely thank the anonymous referee whose comments and suggestions, we believe, have greatly enhanced the accessibility of this article and made the proofs easier to follow. We would also like to thank Prof. Miklós Simonovits for the seminar he gave at the UPC in Barcelona in March 2009, which motivated us to study bipartite Turán numbers.

\section{REFERENCES}

[1] N. Alon, L. Rónyai and T. Szabó, Norm-Graphs: Variations and applications, J. Combin. Theory Ser. B, 76 (1999) 280-290.

[2] T. Bohman and P. Keevash, The early evolution of the $H$-free process, Invent. Math., 181 (2010) 291-336.

[3] B. Bollobás, Extremal Graph Theory, Academic Press, San Diego, 1978. 
[4] W. G. Brown, On graphs that do not contain a Thomsen graph, Canad. Math. Bull., 9 (1966) $281-289$.

[5] P. Erdős, A. Rényi and V. T. Sós, On a problem of graph theory, Studia, Sci. Math. Hungar., 1 (1966) 215-235.

[6] P. Erdős and J. Spencer, Probabilistic Methods in Combinatorics, Academic Press, London, New York, Akadémiai Kiadó, Budapest, 1974.

[7] P. Erdös and A. H. Stone, On the structure of linear graphs, Bull. Amer. Math. Soc., 52 (1946) 1087-1091.

[8] Z. Füredi, An upper bound on Zarankiewicz' problem, Combin. Probab. Comput., 5 (1996) 29-33.

[9] Z. Füredi, New asymptotics for bipartite Turán numbers, J. Combin. Theory Ser. A, 75 (1996) 141144 .

[10] T. Kővári, V. T. Sós and P. Turán, On a problem of K. Zarankiewicz, Colloq. Math., 3 (1954) $50-57$.

[11] V. Pepe, On the algebraic variety $\mathcal{V}_{r, t}$, Finite Fields Appl., 17 (2011) 343-349.

Simeon Ball

Departament de Matemàtica Aplicada IV,

Universitat Politècnica de Catalunya, Jordi Girona 1-3, Mòdul C3, Campus Nord, 08034 Barcelona, Spain

simeon@ma4 . upc . edu

Valentina Pepe

Department of Mathematics, Ghent University, Krijgslaan 281,Building S22

9000 Gent, Belgium

valepepe@cage.ugent.be 